\title{
First passage behaviour of fractional Brownian motion in two-dim- ensional wedge domains
}

\author{
Jae-Hyung Jeon $^{1}{ }^{\text {(a) }}$, Aleksei V. Chechinin ${ }^{2,3}$ and Ralf Metzler ${ }^{1,4}$ (b) \\ 1 Department of Physics, Technical University of Munich, James-Franck Straße, 85747 Garching, Germany \\ ' 2 Akhiezer Institute for Theoretical Physics NSC KIPT, Akademicheskaya Str.1, 61108 Kharkov, Ukraine \\ 3 School of Chemistry, Tel Aviv University, Ramat Aviv, Tel Aviv 69978, Israel \\ 4 Department of Physics, Tampere University of Technology, FI-33101 Tampere, Finland
}

PACS 05.40.-a - Fluctuation phenomena, random processes, noise, and Brownian motion

PACS 02.50.Ey - Stochastic processes

PACS 87.10.Mn - Stochastic modelling

\begin{abstract}
We study the survival probability and the corresponding first passage time density of fractional Brownian motion confined to a two-dimensional open wedge domain with absorbing boundaries. By analytical arguments and numerical simulation we show that in the long time limit the first passage time density scales as $\Theta_{\Theta}(t) \simeq t^{-1+\pi(2 H-2) /(2 \Theta)}$ in terms of the Hurst exponent $H$ and the wedge angle $\Theta$. We discuss this scaling behaviour in connection with the reaction kinetics of FBM particles in a one-dimensional domain.
\end{abstract}

Introduction. - The first passage defines the mo' ment at which a random quantity crosses a given threshold value for the first time. For instance, this could be the time when a random walker first leaves a defined region of space. Other examples include phenomena as diverse as diffusion-limited reactions [1, cyclisation of a polymer 2, 3], stock market dynamics [4, or search problems [5]. The concept of first passage statistics is of quite ubiquitous importance in the theory of stochastic processes [6].

First passage characteristics have been investigated for various geometries. Thus, on semi-infinite domains the probability density of the first passage generally displays a power-law tail, and the associated mean first passage t time diverges [6. Recently, remarkable progress has been achieved, relating the underlying geometry to the resulting first passage behaviour 7 .

In the case of normal diffusion, characterised by the linear growth $\left\langle\mathbf{r}(t)^{2}\right\rangle \simeq t$ of the ensemble-averaged mean squared displacement, the associated first passage problems are obtained by solving the associated diffusion equation with given boundary conditions or, for simpler geometries, by the method of images [6]. For anomalous diffusion with the non-linear growth [9]

$$
\left\langle\mathbf{r}^{2}(t)\right\rangle \simeq t^{2 H}, \quad 2 H \neq 1,
$$

${ }^{(a)}$ E-mail: jae-hyung.jeon@ph.tum.de

(b) E-mail: metz@ph.tum.de of the mean squared displacement, no general theory exists to obtain the corresponding first passage time statistics. Only for certain classes of anomalous diffusion models analytical approaches for the first passage properties are known. Examples include the continuous time random walk model 10] governed by a heavy-tailed waiting time distribution with diverging characteristic waiting time [11 14 or by a power-law jump length distribution with diverging variance (Lévy flights) 15; as well as the diffusion on fractal media 8]. Importantly the first passage time behaviour differs between different stochastic processes despite sharing the same form (1) of the mean squared displacement with dynamic exponent $2 H$.

Fractional Brownian motion (FBM), originally introduced by Kolmogorov [16] and later re-discovered by Mandelbrot and van Ness [17, is a generalised Gaussian process, whose increments in one spatial dimension,

$$
d x(t)=\xi^{H}(t) d t,
$$

are stationary and normally distributed but dependent. Here the quantity $\xi^{H}(t)$ represents fractional Gaussian noise with zero mean $\left(\left\langle\xi^{H}(t)\right\rangle=0\right)$ and autocorrelation

$$
\begin{aligned}
\left\langle\xi^{H}\left(t_{1}\right) \xi^{H}\left(t_{2}\right)\right\rangle & =2 K_{H} H(2 H-1)\left|t_{1}-t_{2}\right|^{2 H-2} \\
& +4 K_{H} H\left|t_{1}-t_{2}\right|^{2 H-1} \delta\left(t_{1}-t_{2}\right) .
\end{aligned}
$$

$K_{H}$ of dimension $\left[K_{H}\right]=\mathrm{cm}^{2} / \mathrm{sec}^{2 H}$ is the anomalous 


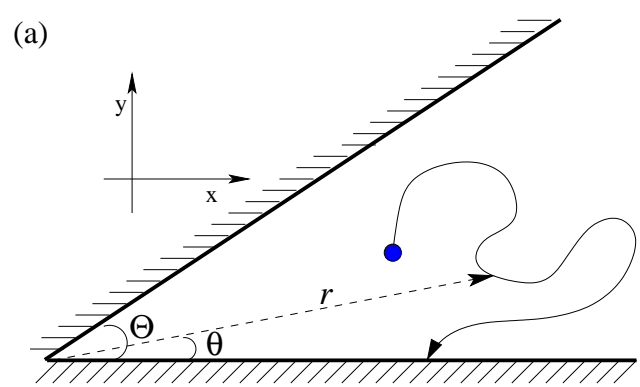

(b)

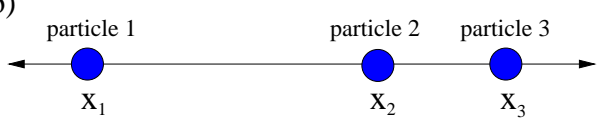

Fig. 1: (a) Stochastic motion of a particle confined to a twodimensional wedge of opening angle $\Theta$. The particle motion motion at time $t$ is described by $\mathbf{r}(t)=(r(t), \theta(t))$ in polar coordinates. The excursion is terminated when the particle hits one of the absorbing walls located at $\theta=0$ or $\Theta$, the moment of first passage. (b) Diffusion-limited reaction of three particles that move according to FBM in one-dimensional space.

diffusion coefficient. The fractional Gaussian noise is negatively (antipersistence) or positively (persistence) correlated for the cases of subdiffusion and superdiffusion, respectively. FBM has been widely used to describe anomalous diffusion phenomena including annual river discharge [18, stock market dynamics [19, the motion of tracer particles in crowded environments 20 23, or single-file diffusion 24. Despite its popularity and deceivingly simple definition in terms of fractional Gaussian noise the exact stochastic properties of FBM are not well understood. The first passage properties are known only for the one-dimensional semi-infinite domain. The associated long-time asymptotic form of the first passage time density $\wp(t) \sim t^{H-2}$ was conjectured by Ding and Yang from scaling arguments 25, and rigorously proved by Molchan 26. Recently, it was shown that the Wilemskii-Fixmann approximation, originally introduced for the description of polymer cyclisation, produces the first passage time behaviour and barrier crossings of particles driven by fractional Gaussian noise [27. The difficulty to analytically access FBM's first passage properties is related to the fact that FBM is a strongly non-Markovian process, which does not fall into the class of semimartingales [28].

Here we analyse the first passage time behaviour of twodimensional FBM confined to a wedge domain of opening angle $\Theta$ (Fig. 1). This problem is of particular interest as the reaction kinetics of three diffusive particles in onedimensional space can be mapped on this problem [6, 29]. In the case of ordinary Brownian motion $(H=1 / 2)$, the wedge problem can be solved exactly using the Green's function formalism [6. Based on analytical and numerical arguments we conjecture the first passage behaviour of FBM for arbitrary Hurst exponent in a wedge geometry.
In the following Section we define multi-dimensional FBM and the first passage in a wedge domain. We then review the Green's function formalism for the Brownian case and obtain analytical forms for the first passage time quantities. We proceed to present our main results, the asymptotic scaling forms of the first passage time behaviour as function of $H$ and $\Theta$. These are numerically confirmed in the subsequent Section. We conclude with a discussion related to reaction kinetics of FBM particles.

Fractional Brownian motion in wedge domain. Consider the random walk of a particle confined to a twodimensional wedge domain of opening angle $\Theta$, see Fig. [1 The particle starts at a position $\mathbf{r}_{0}$ inside the wedge and moves within the domain until eventually it hits one of the absorbing boundaries at a time $t$ for the first time. For this process, we obtain the survival probability

$$
\mathscr{S}_{\Theta}(t)=\int_{W} G(\mathbf{r}, t) d \mathbf{r}
$$

on the wedge domain $W$, and the first passage time density

$$
\wp_{\Theta}(t)=-\frac{d \mathscr{S}(t)}{d t} .
$$

In Eq. (4), $G(\mathbf{r}, t)$ is the probability density to find the particle at position $\mathbf{r}$ at time $t$. Due to the presence of the absorbing boundaries, $G(\mathbf{r}, t)$ is not normalised, as expressed by the survival probability, which decays from $\mathscr{S}_{\Theta}(0)=1$ to $\lim _{t \rightarrow \infty} \mathscr{S}_{\Theta}(t)=0$.

Two-dimensional FBM is defined as a superposition of independent FBMs for each Cartesian coordinate 30 32:

$$
\mathbf{r}(t)=\sum_{i=1}^{2} \int_{0}^{t} d t^{\prime} \xi_{i}^{H}\left(t^{\prime}\right) \hat{x}_{i}+\mathbf{r}_{0},
$$

where $\hat{x}_{i}$ is the unit vector in Cartesian direction $i(i=$ $x, y)$, and $\xi_{i}^{H}(t)$ is the fractional Gaussian noise. Due to this definition it is clear that for a quadrant geometry with $\Theta=\pi / 2$ the absorption to either wall decouples. We now first address the case of normal Brownian motion ( $H=1 / 2)$, for which exact results for the survival and first passage distributions can be found.

First passage process for $H=1 / 2$. - For normal Brownian motion, the diffusion in a wedge domain is described in terms of the Green's function $G(r, \theta ; t)$, that satisfies the diffusion equation in polar coordinates,

$$
\frac{\partial}{\partial t} G(r, \theta ; t)=K_{1 / 2}\left(\frac{\partial^{2}}{\partial r^{2}}+\frac{1}{r} \frac{\partial}{\partial r}+\frac{1}{r^{2}} \frac{\partial^{2}}{\partial \theta^{2}}\right) G(r, \theta ; t) .
$$

The associated boundary conditions are $G(r, 0 ; t)=$ $G(r, \Theta ; t)=0$, representing the absorbing walls. The solution $G$ is completely specified by the initial condition $G(r, \theta ; 0)=\delta\left(r-r_{0}\right) \delta\left(\theta-\theta_{0}\right) / r_{0}$. At long times $t$, the solution $G$ can be approximated by [ 6$]$

$$
G(r, \theta ; t) \simeq \frac{\pi \sin \left(\frac{\pi \theta}{\Theta}\right)}{4 K_{1 / 2} \Theta t} e^{-\left(r^{2}+r_{0}^{2}\right) / 4 K_{1 / 2} t} I_{\pi / \Theta}\left(\frac{r r_{0}}{2 K_{1 / 2} t}\right),
$$


where $I_{\nu}(\mathrm{z})$ is the modified Bessel function of the first kind, which can be expressed by the series expansion

$$
I_{\nu}(z)=(z / 2)^{\nu} \sum_{k=0}^{\infty} \frac{\left(z^{2} / 4\right)^{k}}{k ! \Gamma(\nu+k+1)} .
$$

The survival probability (4) in polar coordinates is then

$$
\mathscr{S}_{\Theta}(t)=\int_{0}^{\Theta} \int_{0}^{\infty} r G(r, \theta ; t) d r d \theta,
$$

from which the property $\mathscr{S}_{\Theta}(0)=1$ follows directly from the sharp initial condition. Using the approximations $I_{\nu}(z) \approx(z / 2)^{\nu} / \Gamma(1+\nu)$ and $e^{-r_{0}^{2} / 4 K_{1 / 2} t} \approx 1$ for the longtime limit in Eq. (8), one obtains the scaling expressions

$$
\begin{aligned}
& \mathscr{S}_{\Theta}(t) \simeq\left(\frac{r_{0}}{\sqrt{K_{1 / 2}}}\right)^{\pi / \Theta} t^{-\pi /(2 \Theta)} \\
& \wp_{\Theta}(t) \simeq \frac{\pi}{2 \Theta}\left(\frac{r_{0}}{\sqrt{K_{1 / 2}}}\right)^{\pi / \Theta} t^{-1-\pi /(2 \Theta)} .
\end{aligned}
$$

Notably, the wedge angle $\Theta$ enters the scaling exponents inverse-proportionally; survival and first passage distributions decay faster for decreasing wedge angle, as it should. We observe an interesting crossover as function of $\Theta$ : as long as $\Theta \geq \pi / 2$ the mean first passage time

$$
\mathcal{T}=\int_{0}^{\infty} t_{\wp_{\Theta}}(t) d t
$$

diverges, $\mathcal{T} \rightarrow \infty$. In particular, in the half-space limit $\Theta=\pi$, the process reduces to the first passage in a onedimensional, semi-infinite geometry (along the $y$-axis), and we find the usual Lévy-Smirnov scaling $\wp(t) \simeq t^{-3 / 2}$. When the wedge angle is smaller, $\Theta<\pi / 2$, the mean first passage time is finite, reflecting the much higher probability to hit one of the two walls of the wedge. Thus, for $\Theta=\pi / 2$, the absorption to either of the two walls can be viewed as two decoupled Brownian random walks in $x$ and $y$ direction. The resulting survival probability is then given as the product of two one-dimensional survival functions corresponding to $\Theta=\pi: \mathscr{S}_{\pi / 2}(t)=\mathscr{S}_{\pi}(t)^{2} \simeq t^{-1}$, for which the mean first passage time is logarithmically divergent. Remarkably, the scaling relation

$$
\mathscr{S}_{\Theta / 2}(t) \simeq \mathscr{S}_{\Theta}^{2}(t)
$$

holds more generally for any $\Theta$, see relations (11).

First passage process for $H \neq 1 / 2$. - Let us now address the case of general Hurst exponent, $H \neq 1 / 2$, for which no analogue to the above Green's function method is known. While there exist dynamic equations for FBM in literature 33,34 , of the form

$$
\frac{\partial}{\partial t} \mathscr{G}(\mathbf{r}, t)=K(t) \nabla^{2} \mathscr{G}(\mathbf{r}, t)
$$

with the time-dependent diffusion coefficient $K(t) \sim$ $t^{2 H-1}$, this description cannot fully specify the behaviour of FBM in the presence of non-natural boundary conditions. To see this, we follow the procedure of the preceding Section, and find the first passage behaviours

$$
\begin{aligned}
\mathscr{S}_{\Theta}^{\mathscr{G}}(t) & \propto t^{-\pi H / \Theta}, \\
\wp_{\Theta}^{\mathscr{C}}(t) & \propto t^{-1-\pi H / \Theta} .
\end{aligned}
$$

While, naturally, the Brownian case $H=1 / 2$ is consistent with the results of the previous Section, the results (15) are inconsistent with Molchan's result $\wp(t) \simeq t^{H-2}$ for a completely open wedge, $\Theta=\pi$. Therefore, for arbitrary wedge angle $\Theta$ and Hurst exponent $H$ the distributions (15) cannot be correct. In fact, the same inconsistency is found when one naively applies the method of images to the free space solution $\left(4 \pi K_{H} t^{2 H}\right)^{-1 / 2} \exp \left(-x^{2} /\left[4 K_{H} t^{2 H}\right]\right)$.

We now argue in favour of a conjecture for the correct scaling forms of the first passage time quantities. Let us start by recollecting the known special cases, for which analytical results are available:

(i) When $\Theta=\pi$, the first passage time quantities must converge to Molchan's result for a one-dimensional semiinfinite domain [26]:

$$
\begin{aligned}
\mathscr{S}_{\pi}(t) & \simeq t^{H-1}, \\
\wp_{\pi}(t) & \simeq t^{H-2},
\end{aligned}
$$

valid in the long time limit $t \gg 1$.

(ii) When $\Theta=\pi / 2$, due to the independence of $x$ and $y$ motion the survival probability, by above argument, is given as the product of two one-dimensional survival probabilities, $\mathscr{S}_{\pi / 2}=\mathscr{S}_{\pi}^{2}$, and thus

$$
\begin{aligned}
\mathscr{S}_{\pi / 2}(t) & \simeq t^{2 H-2}, \\
\wp_{\pi / 2}(t) & \simeq t^{2 H-3} .
\end{aligned}
$$

(iii) For $H=1 / 2$, we know the analytical results (11) with the full $\Theta$-dependence.

Assuming that the first passage time exponents are simple combinations of the Hurst exponent $H$ and the wedge angle $\Theta$, to satisfy the above three special cases we conjecture the unique scaling form

$$
\begin{aligned}
\mathscr{S}_{\Theta}(t) & \simeq t^{\pi(2 H-2) /(2 \Theta)}, \\
\wp_{\Theta}(t) & \simeq t^{-1+\pi(2 H-2) /(2 \Theta)} .
\end{aligned}
$$

These results imply that the survival decays faster for slower diffusion (i.e., lower value of $H$ ), in analogy with Molchan's result and findings for the barrier crossing of FBM 27. Note that the inconsistent results (15) based on the dynamic equation (14) follow from our results through the substitution $H \rightarrow 1-H$.

Simulations. - To numerically verify the conjecture (18) for the survival probability and the first passage time density, we performed simulations for various wedge angles and Hurst exponents, and compared the results with 

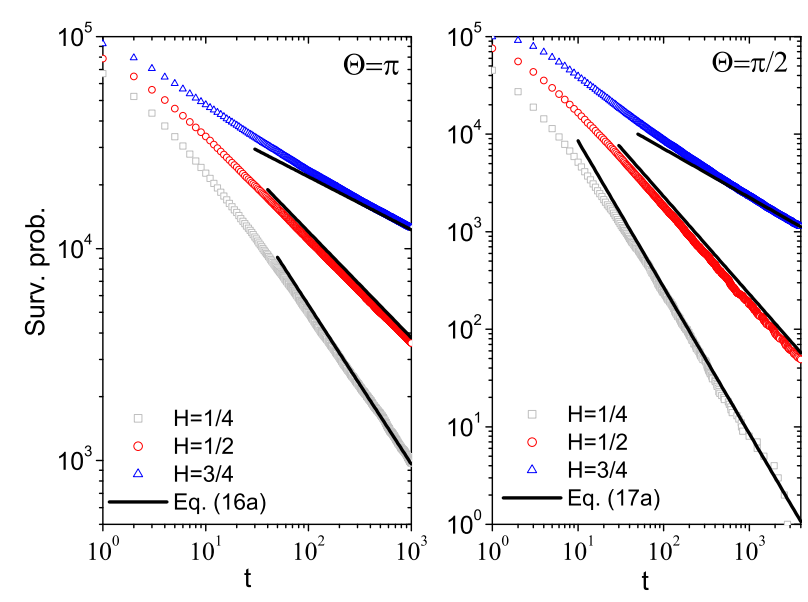

Fig. 2: Survival probability $\mathscr{S}_{\Theta}(t)$ for wedge angles $\Theta=\pi$ (Left) and $\Theta=\pi / 2$ (Right) with the Hurst exponents $H=1 / 4$, $1 / 2$, and $3 / 4$ (from bottom to top). The lines represent the expected scaling behaviours, Eqs. 116a and (17a).

Eqs. (18). For this comparison, we here focus on the survival probability, which generally shows better statistics.

Our simulations procedure is as follows. A wedge of opening angle $\Theta$ is constructed by imposing two absorbing walls at $y=0$ and $y=(\tan \Theta) x$ in the $x$-y plane (Fig. 1(a)). At $t=0$, the particle is located at $\mathbf{r}_{0}=$ $\left(r_{0} \cos \frac{\Theta}{2}, r_{0} \sin \frac{\Theta}{2}\right)$ on the line bisecting the wedge. For $t>0$, the particle undergoes two-dimensional FBM in discrete time indexed by $n$, following the rule

$$
\begin{aligned}
& \mathbf{r}\left(t_{n}\right)= \\
& \left(\sum_{m=1}^{n} \xi_{1}^{H}\left(t_{m}\right)+r_{0} \cos \frac{\Theta}{2}, \sum_{m=1}^{n} \xi_{2}^{H}\left(t_{m}\right)+r_{0} \sin \frac{\Theta}{2}\right) .
\end{aligned}
$$

Here, the two independent fractional Gaussian noise functions $\xi_{1}^{H}(t)$ and $\xi_{2}^{H}(t)$ are generated by the Hosking method 31, 35. The simulation is terminated when the particle escapes the wedge domain for the first time. This is the survival time for the particle in the given run. From repeated runs we construct the survival probability $\mathscr{S}_{\Theta}(t)$ as the accumulated number of particles surviving until time $t$. For each given wedge angle and Hurst exponent, the survival probability $\mathscr{S}_{\Theta}(t)$ was obtained from 100,000 runs and for initial radius $r_{0}=0.25$.

To confirm that our simulations procedure produces correct results for the survival probability, we first consider the two special cases of wedge angles $\Theta=\pi$ and $\pi / 2$, and compare them to the predicted scaling behaviours (16a) and (17a). Fig. 2 shows on the left the distributions of $\mathscr{S}_{\Theta}(t)$ obtained from simulations of a fully open wedge, $\Theta=\pi$, for Hurst exponents $H=1 / 4$ (subdiffusion), $1 / 2$ (normal diffusion), and 3/4 (superdiffusion). Clearly, the distributions follow the predicted scaling behaviours for this case corresponding to the one-dimensional, semiinfinite domain. Similarly, for the case of a rectangular wedge $(\Theta=\pi / 2)$ on the right of Fig. 2 we find excellent verification of our simulations method, compared to the predicted behaviour $\mathscr{S}_{\pi / 2}(t) \sim t^{2 H-2}$ for all values of $H$.

We now investigate whether our conjecture (18a) for the survival probability also holds for other wedge angles. In our simulation, the survival probabilities for wedge angles $\Theta=\pi / 4,3 \pi / 4,5 \pi / 4$, and $7 \pi / 4$ were investigated, for Hurst exponents $H=1 / 4,1 / 2$, and $3 / 4$. As demonstrated in Fig. 3. the simulated survival probabilities remarkably well follow our conjecture (18a) for all wedge angles and Hurst exponents. Particularly, the scaling exponents of the survival indeed decreases for growing values of the Hurst exponent $H$, in line with Molchan's result and findings for the barrier crossing behaviour of FBM.

Discussion. - We studied the first passage properties of two-dimensional FBM confined to a wedge domain with absorbing boundaries. Starting from special cases, for which exact long-time scaling expressions can be analytically derived, we obtained functional dependencies of first passage time exponents on the Hurst exponent $H$ and wedge angle $\Theta$. By numerical analysis we confirmed this conjecture. We believe that our results are an important additional brick in the construction of a clear picture of the elusive FBM process. The obtained forms (18) of the first passage time quantities imply that the mean first passage time of a particle from a wedge domain across the domain walls, $\mathscr{T}$, diverges for wedge angles $\Theta \geq \Theta_{c}$. The critical angle corresponds to $\Theta_{c}=\pi(1-H)$.

From the above results one may obtain some insight into the reaction kinetics of three particles in one-dimensional space, driven by FBM (Fig. 1(b)): (1) Surrounded prey. Let us consider the diffusion-limited reaction of the central particle with either of the two comrade particles. The problem can be transformed such that one particle is confined between two moving absorbing boundaries [6]. If $x_{i}(t)$ denotes the position of the $i$ th particle at time $t$, the survival condition of the central particle is $x_{1}(t)<x_{2}(t)$ and $x_{2}(t)<x_{3}(t)$ for particles sharing the same diffusion properties $\left(H\right.$ and $\left.K_{H}\right)$. We regard $x_{i}(t)$ as the $i$ th coordinate of a single particle, that diffuses in three-dimensional space. Then, the survival criterion is understood such that this particle diffuses inside a domain limited by absorbing planes at $x_{1}=x_{2}$ and $x_{3}=x_{2}$. This is equivalent to the first passage problem for two-dimensional motion in a wedge domain, the wedge angle given by the two intersecting planes, i.e., $\Theta_{\text {mid }}=\pi / 3$ (see Ref. [6]). Thus, from the distribution (18a) we find that the survival probability for the central particle has the asymptotic form $\mathscr{S}_{\text {mid }}(t) \sim t^{3(H-1)}$. Contrary to our naive expectation, the above scaling exponent shows that superdiffusive particles (with $H>1 / 2$ ) survive longer than ordinary Brownian particles $(H=1 / 2)$, and subdiffusive particles have a better chance to meet each other. These characteristics will lead to significant differences in the total reaction amount, compare Figs. 2 and 3. Intriguingly the mean reaction time $\mathscr{T}$ is finite for $H<H^{*}=2 / 3$, meaning that 

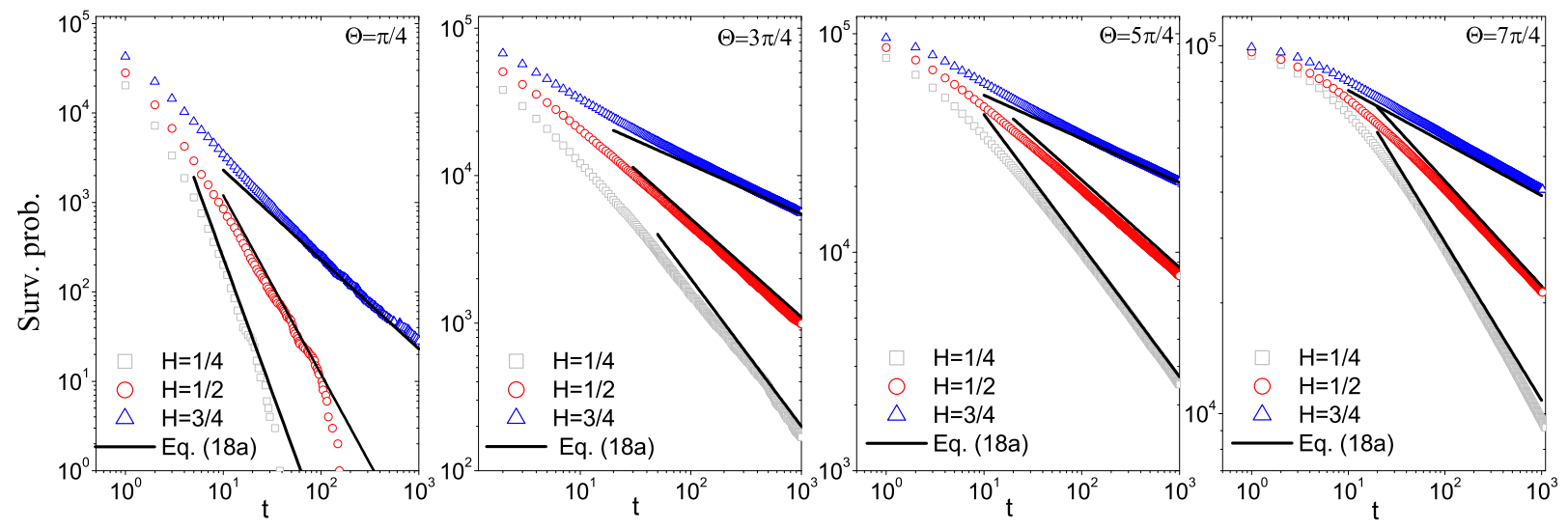

Fig. 3: Survival probability $\mathscr{S}_{\Theta}(t)$ for wedge angles $\Theta=\pi / 4,3 \pi / 4,5 \pi / 4$, and $7 \pi / 4$, with Hurst exponents $H=1 / 4,1 / 2$, and $3 / 4$ (from bottom to top). The corresponding expected scaling behaviours (cf. Eq. (18a)) are depicted by the full lines.

reactions always occur for subdiffusive motion, similar to the considerations in Ref. [36. (2) Chased prey. For the case that one of the corner particles $\left(x_{3}\right.$, say) is chased by the two other diffusing particles $\left(x_{1}\right.$ and $\left.x_{2}\right)$, the problem is reformulated to the task of finding the survival probability for the wedge domain of angle $\Theta_{\text {end }}=2 \pi / 3$. Hence for identical particles the probability that one corner particle survives until time $t$ scales as $\mathscr{S}_{\text {end }}(t) \sim t^{3(H-1) / 2}$. In this case the reaction is slower than that of the surrounded particle. Note the scaling relation $\mathscr{S}_{\text {mid }}(t) \simeq \mathscr{S}_{\text {end }}^{2}(t)$, such that the reaction of a particle surrounded by two others corresponds to the product of two independent corner particle reactions.

We note that closer inspection of the simulated survival probabilities shows that in the non-Markovian case $(H \neq 1 / 2)$ the first passage process exhibits a somewhat intricate scaling behaviour: the long-time behaviours (18) are preceded by another distinct scaling law at intermediate times. This behaviour is particularly noticeable for the cases of wedge angle $\Theta \leq \pi$, for which first passage events occur easily at short and intermediate times. The apparent intermediate scaling behaves differently, depending on the diffusion characteristics. For subdiffusion, the intermediate scaling exponent is smaller than the long-time exponent $\frac{\pi}{\Theta}(1-H)$ and larger for superdiffusion.

The first passage behaviour displayed by FBM is different from results derived from the diffusion equation with time-dependent diffusion coefficient. Latter would lead to an inconsistent $H$-dependence, corresponding to the replacement $H \rightarrow 1-H$ in the correct forms (18). Similar inconsistency occurs if the method of images were applied to construct the solution in the presence of non-natural boundary conditions. We note in passing that the substitution $H \rightarrow 1-H$ in our results for $\Theta=\pi$ leads to the scaling for the first passage behaviour found in the analysis of a generalised Langevin equation in Ref. 37.

The somewhat counterintuitive behaviour that smaller $H$ implies faster decay of the first passage time density, may be connected to the fact that FBM is fuelled by external noise, that is not balanced by friction. FBM, that is, does not obey the fluctuation-dissipation relation, in contrast to the generalised (fractional) Langevin equation [33, 34, 38 that describes FBM in conditions close to thermal equilibrium, when the fluctuation-dissipation theorem is valid. We will investigate the latter behaviour in a separate work.

We finally note that while for a compact process in one dimension the first passage is identical to the first arrival to a given position, these processes are no longer necessarily equal to each other in higher dimensions. This may also be the reason why the Wilemskii-Fixman approximation reproduces Molchan's result $\wp(t) \simeq t^{H-2}$ in one dimension, but delivers a different result in higher dimensions.

We thank Michael Lomholt and Igor Sokolov for helpful discussion. Financial support from the Academy of Finland (FiDiPro scheme) and the European Commission through MC IIF Grant No. 219966 LeFrac is gratefully acknowledged.

Appendix. - The survival probabilities presented in Fig. 3 were obtained with the same initial starting point and number of simulation runs for consistency. Depending on the wedge angle and Hurst exponent, however, some cases are more problematic to show reliable long-time scaling properties within the time window we used. Here we present supplementary results for these cases.

(i) Fig. 4 depicts the case for $\Theta=\pi / 4$ with $r_{0}=2.0$ (i.e., eight times the value used in the main text) and 250,000 


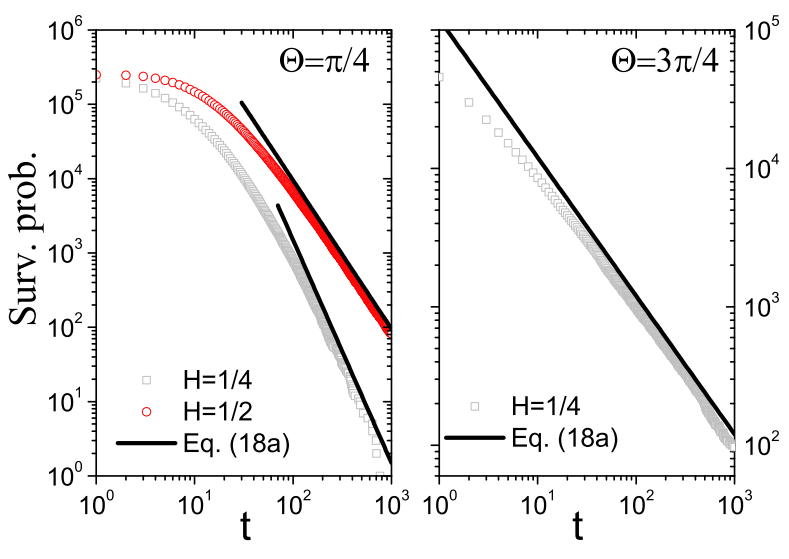

Fig. 4: (Left) Survival probability $\mathscr{S}_{\Theta}(t)$ for wedge angle $\Theta=$ $\pi / 4$ and Hurst exponents $H=1 / 4$ (below) and $1 / 2$ (above). Here $r_{0}=2.0$, and the number of simulation runs is 250,000 . (Right) Survival probability $\mathscr{S}_{\Theta}(t)$ at wedge angles $\Theta=3 \pi / 4$ for the Hurst exponent $H=1 / 4$. Here $r_{0}=0.10$, and number of simulation runs is 100,000. The solid lines are the expected power-laws predicted by Eq. (18a).

runs. Now the survival probabilities for $H=1 / 4$ and $1 / 2$ exhibit satisfactory long-time scaling.

(ii) To obtain extended long-time scaling behaviour for $H=1 / 4$ and wedge angle $\Theta=3 \pi / 4$, the initial distance from the origin was decreased to $r_{0}=0.10$. The obtained result in Fig. 4 shows good agreement with Eq. (18a).

\section{REFERENCES}

[1] von Smoluchowski M., Phys. Z., 17 (1916) 557.

[2] Sokolov I. M., Phys. Rev. Lett., 90 (2003) 080601.

[3] Likthman A. E. and Marques C. M., Europhys. Lett., 75 (2006) 971.

[4] Bouchaud J.-P. and Potters M., Theory of Financial Risk and Derivative Pricing: From Statistical Physics to Risk Management (Cambridge University Press, Cambridge, UK) 2003.

[5] Lomholt M. A., Koren T., Metzler R and Klafter J., Proc. Natl. Acad. Sci. USA, 105 (2008) 11055.

[6] Redner S., A Guide to First-passage Processes (Cambridge University Press, Cambridge, UK) 2007.

[7] Bénichou O., Chevalier C., Klafter J, Meyer B. and Voituriez R., Nature Chem., 2 (2010) 472; Condamin S., Bénichou O. and Moreau M., Phys. Rev. Lett., 95 (2007) 260601.

[8] Condamin S., Bénichou O., Tejedor V., Voituriez R. and Klafter J., Nature, 450 (2007) 77.

[9] Metzler R. and Klafter J., Phys. Rep., 339 (2000) 1; J. Phys. A, 37 (2004) R161.

[10] Scher H. and Montroll E. W., Phys. Rev. B, 12 (1975) 2455; Montroll E. W. and Weiss G. H., J. Math. Phys., 10 (1969) 753.

[11] Metzler R. and Klafter J., Physica A, 278 (2000) 107.
[12] Rangarajan G. and Ding M., Phys. Rev. E, 62 (2000) 120; Phys. Lett. A, 273 (2000) 322.

[13] Condamin S., Bénichou O. and Klafter J., Phys. Rev. Lett., 98 (2007) 250602.

[14] Condamin S., Tejedor V., Voituriez R., BÉnichou O. and Klafter J., Proc. Nat. Aca. Sci. USA, 105 (2008) 5675.

[15] Chechion A. V., Metzler R., Gonchar V. Y., Klafter J. and Tanatarov L. V., J. Phys. A, 36 (2003) L537.

[16] Kolmogorov A. N., Dokl. Acad. Sci. USSR, 26 (1940) 115.

[17] Mandelbrot B. B. and van Ness J. W., SiAM Rev., 1 (1968) 422.

[18] Hurst H. E., Trans. Am. Soc. Civ. Eng., 116 (1951) 770; Hurst H. W., Black R. O. and Simaika Y. M., Long Term Storage: An Experimental Study (Constable, London, UK) 1965.

[19] Simonsen I., Physica A, 322 (2003) 597; Frangos N. E., Vrontos S. D. and Yannacopoulos A. N., Appl. Stoch., Models Bus. Ind., 23 (2007) 403.

[20] Szymanski J. and Weiss M., Phys. Rev. Lett., 103 (2009) 038102.

[21] Magdziarz M., Weron A., Burnecki K. and Klafter J., Phys. Rev. Lett., 103 (2009) 180602; Magdziarz M. and Klafter J.,Phys. Rev. E822010011129.

[22] Jeon J.-H., Tejedor V., Burov S. Barkai E., Selhuber-Unkel C., Berg-Sørensen K., Oddershede L. and Metzler R., Phys. Rev. Lett., 106 (2011) 048103.

[23] Weber S. C., Spakowitz A. J. and Theriot J. A., Phys. Rev. Lett., 104 (2010) 238102.

[24] Lizana L. and Ambjörnsson T., Phys. Rev. Lett., 100 (2008) 200601; Lizana L., Ambjörnsson T., Taloni A., Barkai E. and Lomholt M. A., Phys. Rev. E, 81 (2010) 051118.

[25] Ding M. and Yang W., Phys. Rev. E, 52 (1997) 207.

[26] Molchan G. M., Commun. Math. Phys., 205 (1999) 97.

[27] Sliusarenko O. Y., Gonchar V. Y., Chechkin A. V., Sokolov I. M. and Metzler R., Phys. Rev. E, 81 (2010) 041119.

[28] Weron A. and Magdziarz M., EPL, 86 (2009) 60010.

[29] Ben-Avraham D., J. Chem. Phys., 88 (1988) 941.

[30] Yaglom A., Correlation Theory of Stationary and Related Random Functions, Vol. I (Springer-Verlag, Berlin) 1987.

[31] Jeon J.-H. and Metzler R., Phys. Rev. E, 81 (2010) 021103.

[32] Unterberger J., Ann. Prob., 37 (2009) 565.

[33] Lutz E., Phys. Rev. E, 64 (2001) 051106.

[34] Wang K. G. and Lung C. W., Phys. Lett. A, 151 (1990) 119.

[35] Hosking J. R. M., Water Resour. Res., 20 (1984) 1989.

[36] Guigas G. and Weiss M., Biophys. J., 94 (2008) 90.

[37] Taloni A., Chechkin A. V. and Klafter J., Phys. Rev. Lett., 104 (2010) 160602.

[38] Goychuk I., Phys. Rev. E, 80 (2009) 046125. 